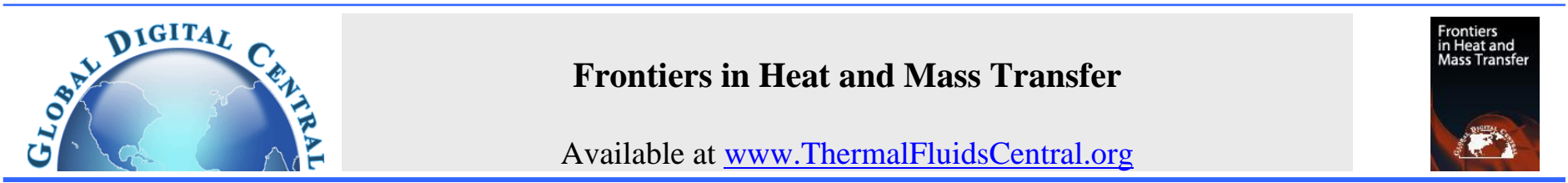

\title{
STEADY MHD FLOW OVER A YAWED CYLINDER WITH MASS TRANSFER
}

\author{
A. Sahaya Jenifer ${ }^{\mathrm{a}}$, P. Saikrishnan ${ }^{\mathrm{a}, *}$, J. Rajakumar ${ }^{\mathrm{b}}$ \\ ${ }^{a}$ Department of Mathematics, National Institute of Technology, Tiruchirappalli, India \\ ${ }^{\mathrm{b}}$ Department of Mathematics, Government College of Engineering, Tirunelveli, India
}

\begin{abstract}
This paper examines the steady magnetohydrodynamic (MHD) flow of water over a yawed cylinder with variable fluid properties and non-uniform mass transfer. The impact of viscous dissipation is taken into consideration. The velocity and temperature fields are governed by coupled nonlinear partial differential equations together with boundary constraints. These governing equations are converted to dimensionless form with suitable non-similar transformations and then solved using an implicit finite difference method and the quasi-linearization technique. The results indicate that the yaw angle enhancement declines the skin friction coefficient in the axial direction and the heat transfer coefficient. It is also ascertained that the separation can be delayed by enhancing the MHD effect, the suction parameter with slot movement in the downstream direction.
\end{abstract}

Keywords: Boundary layer flow, Magnetohydrodynamic, Non-uniform mass transfer, Non-similar, Variable viscosity

\section{INTRODUCTION}

Magnetohydrodynamic heat and mass transfer have numerous applications in magnetohydrodynamic electrical power generation, boundary layer control, MHD generators, MHD pumps, petroleum industries, plasma studies and many others. Many methods have been evolved to control the boundary layer's behavior, and out of that, the enhancement of the MHD principle plays a crucial part in altering the boundary layer's structure and thus influencing flow in the intended direction. The mutual interaction of electromagnetic field and fluid velocity characterize the MHD boundary layer flow. Aldoss et al. (1996) have investigated analytically and numerically the effect of MHD mixed convection flow over a horizontal circular cylinder. El-Amin (2003) has studied the effect of MHD forced convection over a non-isothermal horizontal cylinder embedded in a fluid-saturated porous medium. Nagaraju et al. (2019); Kumar et al. (2021) analyzed the MHD flow and heat transfer with geometries of the circular horizontal pipe and porous disks, respectively.

The geometry considered here is an infinite yawed circular cylinder due to its extensive engineering design applications, yet only a handful of investigations have been made so far. Several authors have published experimental results (King, 1977; Ramberg, 1983; Thakur et al., 2004; Mityakov et al., 2017) and numerical results (Marshall, 2003) of flow past a yawed cylinder. Recently, Patil et al. (2020) have published a work on mixed convection flow past a yawed cylinder. However, no investigation is found on MHD flow past a yawed cylinder so far.

Boundary layer is non-similar in nature. This non-similarity occurs due to the body's curvature or the velocity profiles at the edge or due to the surface mass transfer or perhaps an amalgamation of all the abovementioned factors. A brief review on obtaining non-similar solutions and the references of apposite works are found by Dewey and Gross (1967). Non-similar solutions for a compressible laminar boundary-layer flow had been obtained by Davies and Walker (1977) using the finite difference method, and their behavior was presented near the point of separation. Venkatachala and Nath (1980) have brought non-similar solutions using the finite difference method for a boundary layer flow of steady laminar incompressible two-dimensional and axisymmetric porous bodies with pressure gradient. Roy (2001) shows non-similar solutions for a compressible flow over the yawed cylinder using the implicit finite difference method along with the quasi-linearization technique. Subhashini and Samuel (2016) obtained a non-similar solution of steady compressible flow over a thin cylinder.

Fundamental physical properties of fluid often change notably with temperature; hence it is essential to acknowledge such properties as a function of temperature. Several investigators have studied the impact of variable fluid properties for steady laminar flows over different heated surfaces (Chin et al., 2007; Eisenhuth and Hoffman, 1981; Pantokratoras, 2005; Mohammad, 2020).

Surface mass transfer has a substantial impact in preventing or slowing down the boundary layer separation. The influence of non-uniform mass transfer on water flow past various bodies has been studied by Saikrishnan and Roy (2003a,b), and it is discovered that non-uniform suction delays the point of separation. Tashtoush et al. (2000) have investigated the mass transfer's effect of non-Newtonian fluid on a power-law stretched surface. Researchers Ponnaiah (2012); Revathi et al. (2014); Roy and Saikrishnan (2004) have solved steady/unsteady forced convection flow problem over a yawed cylinder taking non-uniform slot suc-

*Corresponding author. Email: psai@nitt.edu 
tion/injection into consideration. Ganapathirao et al. (2019); Jenifer et al. (2021) analyzed the effects of non-uniform slot suction/injection on MHD mixed convective flow past a vertical wedge and sphere, respectively.

The current study is concentrated on the effects of MHD flow over a yawed cylinder with non-uniform mass transfer and temperature-dependent viscosity and Prandtl number. The fluid considered here is water due to its extreme practical applications.

\section{MATHEMATICAL FORMULATION}

The flow model of the steady laminar MHD past a yawed infinite cylinder of radius $R$, set in a free stream with the oncoming free stream velocity $U_{\infty}$ (see Fig. 1). A constant magnetic field $B_{0}$ is applied in the normal direction to the cylinder's surface. The surface of a yawed cylinder and the free stream are at constant temperatures of $T_{w}, T_{\infty}$, respectively.

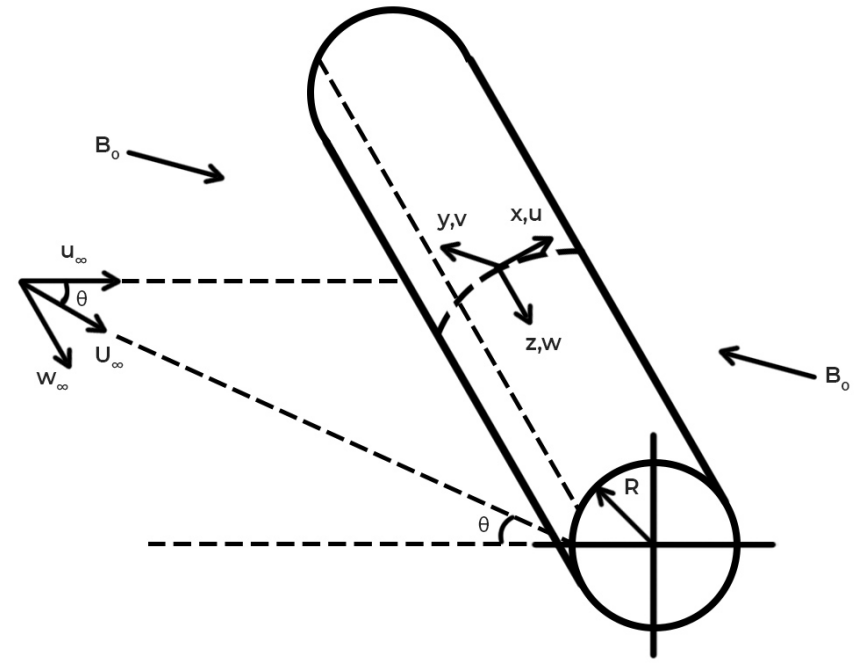

Fig. 1 Flow model

The variation in temperature between the sphere's surface and the free stream is taken in the range $0^{\circ} \mathrm{C}-40^{\circ} \mathrm{C}$. The viscosity $(\mu)$ and thermal conductivity $(k)$ show a significant variation with temperature, and so does the Prandtl number $(P r)$. So, both $\mu$ and $\operatorname{Pr}$ can be expressed as inverse linear functions of $T$ as given by Eswara and Nath (1994).

$$
\mu=\frac{1}{(a+b T)} \quad \text { and } \quad \operatorname{Pr}=\frac{1}{(c+d T)}
$$

where

$$
a=53.41, \quad b=2.43, \quad c=0.068, \quad d=0.004
$$

Within this temperature limit considered, fluid density $(\rho)$ and specific heat $\left(c_{p}\right)$ vary with the temperature only up to a maximum of $1 \%$. This minute variation allows us to take $\rho$ and $c_{p}$ as constants.

The governing equations are:

$$
\begin{gathered}
(r u)_{x}+(r v)_{y}=0 \\
u u_{x}+v u_{y}=u_{e}\left(u_{e}\right)_{x}+\frac{1}{\rho}\left(\mu u_{y}\right)_{y}-\frac{\sigma B_{0}^{2}}{\rho}\left(u-u_{e}\right) \\
u w_{x}+v w_{y}=\frac{1}{\rho}\left(\mu w_{y}\right)_{y}-\frac{\sigma B_{0}^{2}}{\rho} w \\
u T_{x}+v T_{y}=\frac{1}{\rho}\left(\frac{\mu}{P r} T_{y}\right)_{y}+\frac{\mu}{\rho c_{p}}\left(u_{y}^{2}+w_{y}^{2}\right)+\frac{\sigma B_{0}^{2}}{\rho c_{p}}\left(u^{2}-u_{e} u\right)
\end{gathered}
$$

Boundary conditions:

$$
u(x, 0)=0, \quad u(x, \infty)=u_{e}(x), \quad v(x, 0)=v_{w}(x),
$$

$w(x, 0)=0, \quad w(x, \infty)=w_{e}(x), \quad T(x, 0)=T_{w}, \quad T(x, \infty)=T_{\infty}$

The transformations to non-dimensionalize the Eqs. (3) - (6) are,

$$
\begin{gathered}
\psi(x, y)=R u_{\infty}\left(\frac{2 \xi}{R e}\right)^{1 / 2} f(\xi, \eta), \quad u=\psi_{y}, \quad v=-\psi_{x} \\
\xi=\int_{0}^{x} \frac{u_{e}}{u_{\infty}} d\left(\frac{x}{R}\right), \quad \eta=\left(\frac{u_{e}}{u_{\infty}}\right)\left(\frac{R e}{2 \xi}\right)^{1 / 2}\left(\frac{y}{R}\right) \\
G=\frac{T-T_{\infty}}{T_{w}-T_{\infty}}, \quad u_{\infty}=U_{\infty} \cos \theta, \quad R e=\frac{u_{\infty} R}{\nu}
\end{gathered}
$$

The above transformations satisfy Eq. (3) identically the non-dimensional form of the Eqs. (4), (5) and (6) are given below.

$$
\begin{gathered}
\left(N F_{\eta}\right)_{\eta}+f F_{\eta}+\beta\left(1-F^{2}\right)+M P(1-F)=2 \xi\left(F F_{\xi}-f_{\xi} F_{\eta}\right) \\
\left(N S_{\eta}\right)_{\eta}+f S_{\eta}-M P S=2 \xi\left(F S_{\xi}-f_{\xi} S_{\eta}\right) \\
\left(\frac{1}{P r} N G_{\eta}\right)_{\eta}+f G_{\eta}+N E c\left[\left(\frac{u_{e}}{u_{\infty}}\right)^{2} \cos ^{2} \theta F_{\eta}^{2}+\sin ^{2} \theta S_{\eta}^{2}\right] \\
+P E c M\left(\frac{u_{e}}{u_{\infty}}\right)^{2} \cos ^{2} \theta F(F-1)=2 \xi\left(F G_{\xi}-f_{\xi} G_{\eta}\right)
\end{gathered}
$$

with the boundary conditions

$$
\begin{aligned}
F(\xi, 0) & =0, & F(\xi, \infty) & =1 \\
S(\xi, 0) & =0, & & S(\xi, \infty)=1 \\
G(\xi, 0) & =1, & G(\xi, \infty) & =0
\end{aligned}
$$

where

$$
\begin{gathered}
N=\frac{\mu}{\mu_{\infty}}=\frac{a+b T_{\infty}}{a+b T}=\frac{1}{1+E_{1} G} \\
E_{1}=\frac{b \Delta T_{w}}{a+b T_{\infty}} \\
\operatorname{Pr}=\frac{1}{c+d T}=\frac{1}{E_{2}+E_{3} G}, \\
E_{2}=c+d T_{\infty}, \quad E_{3}=d \Delta T_{w}, \\
\Delta T_{w}=T_{w}-T_{\infty}, \quad \frac{u}{u_{e}}=f_{\eta}=F, \\
E c=\frac{U_{\infty}^{2}}{c_{p}\left(T_{w}-T_{\infty}\right)}, \quad \beta(\xi)=\frac{2 \xi}{u_{e}} \frac{d u_{e}}{d \xi}, \\
P=3 \xi\left(\frac{u_{\infty}}{u_{e}}\right)^{2}, \quad M=\frac{2}{3} \frac{\sigma B_{0}^{2} R}{\rho u_{\infty}} \\
\nu_{\infty}=\frac{\mu_{\infty}}{\rho} \\
f=\int_{0}^{\eta} F d \eta+f_{w}
\end{gathered}
$$

where

$$
f_{w}=-(2 \xi)^{-1 / 2}(R e)^{1 / 2} \int_{0}^{x} \frac{v_{w}(x)}{u_{\infty}} d\left(\frac{x}{R}\right)
$$

The free stream velocity components in chordwise and spanwise directions are given by

$$
\begin{aligned}
& u_{e}=2 u_{\infty} \sin \bar{x}, \quad u_{\infty}=U_{\infty} \cos \theta \\
& w_{e}(\bar{x})=w_{\infty}=U_{\infty} \sin \theta, \quad \bar{x}=\frac{x}{R}
\end{aligned}
$$

$\xi, \beta(\xi)$ and $P(\xi)$ can be written as expressions in $\bar{x}$ as follows.

$$
\xi=2 P_{1}(\bar{x}), \quad \beta=\frac{2 \cos \bar{x}}{P_{2}}, \quad P=\frac{3}{2 P_{2}}
$$




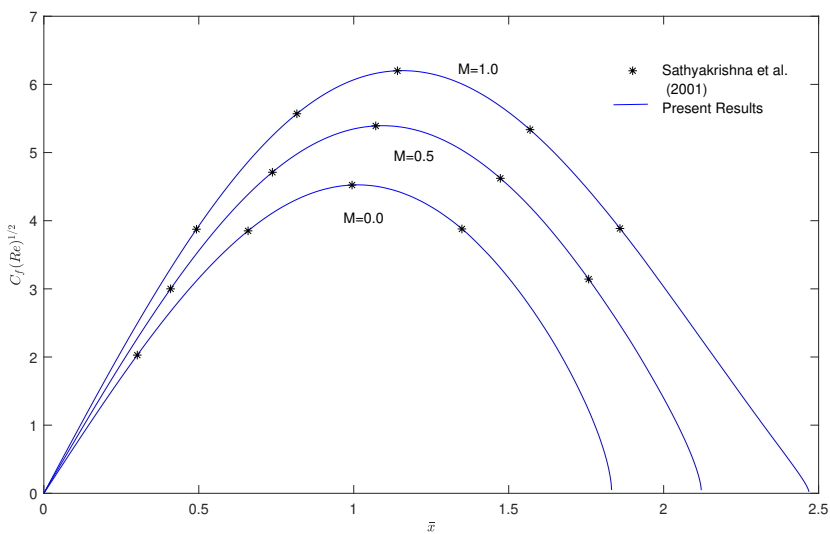

Fig. 2 Comparison of the effect of MHD parameter on the skin friction coefficient for constant fluid properties with those of Sathyakrishna et al. (2001) where $T_{\infty}=18.7^{\circ} \mathrm{C}, \Delta T_{w}=10.0^{\circ} \mathrm{C}, \theta=0^{\circ}$
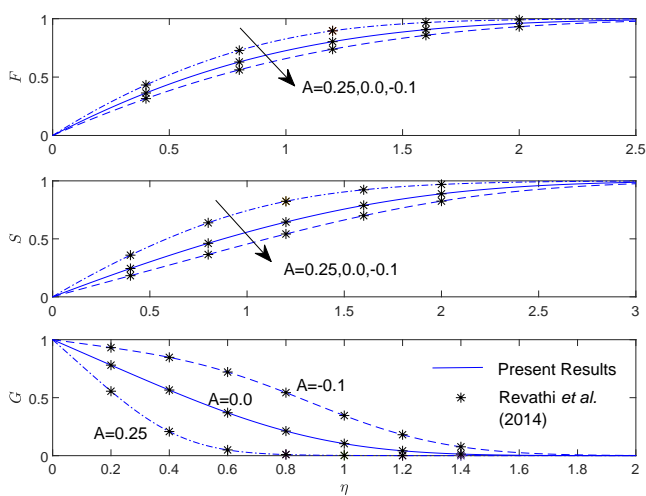

Fig. 3 Comparison of the effect of mass transfer parameter on the velocity and temperature profiles for variable fluid properties with those of Revathi et al. (2014) where $M=0.0, \theta=30^{\circ}$

where

$$
P_{1}=1-\cos \bar{x}, \quad \text { and } \quad P_{2}=1+\cos \bar{x}
$$

$f_{w}$ is given by

$$
f_{w}= \begin{cases}0, & \bar{x} \leq \bar{x}_{0} \\ A\left(P_{1}\right)^{-1 / 2}\left[1-\cos \left(\omega^{*}\left(\bar{x}-\bar{x}_{0}\right)\right)\right], & \bar{x} \in\left[\bar{x}_{0}, \bar{x}_{0}^{*}\right] \\ A\left(P_{1}\right)^{-1 / 2}\left[1-\cos \left(\omega^{*}\left(\bar{x}_{0}^{*}-\bar{x}_{0}\right)\right)\right], & \text { otherwise }\end{cases}
$$

Also, $v_{w}$ is considered to be

$$
v_{w}=\left\{\begin{array}{l}
-2 A u_{\infty}(R e)^{-1 / 2} \sin \left(\omega^{*}\left(\bar{x}-\bar{x}_{0}\right)\right), \quad \text { where } \bar{x} \in\left[\bar{x}_{0}, \bar{x}_{0}^{*}\right] \\
0, \text { otherwise }
\end{array}\right.
$$

It is suitable to write the equations in $\bar{x}$ instead of $\xi$.

$\bar{x}$ and $\xi$ are related by

$$
\xi \frac{\partial}{\partial \xi}=Q(\bar{x}) \frac{\partial}{\partial \bar{x}}
$$

where,

$$
Q(\bar{x})=\tan \frac{\bar{x}}{2}
$$

Substituting Eqs. (18) and (19) in the Eqs. (9), (10) and (11), we obtain the dimensionless equations,

$$
\left(N F_{\eta}\right)_{\eta}+f F_{\eta}+\beta\left(1-F^{2}\right)+M P(1-F)=2 Q(\bar{x})\left(F F_{\bar{x}}-f_{\bar{x}} F_{\eta}\right)
$$

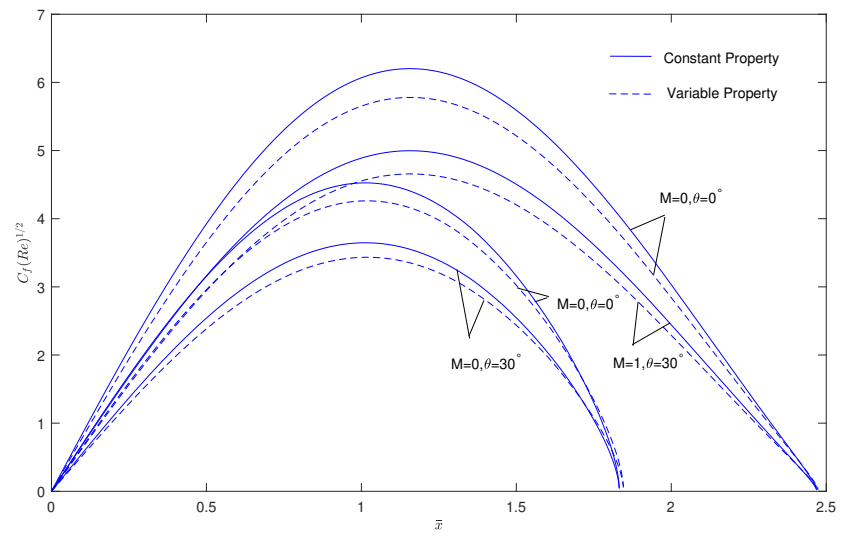

Fig. 4 Effect of the constant and variable properties on the skin friction coefficient $\left(C_{f}(R e)^{1 / 2}\right)$

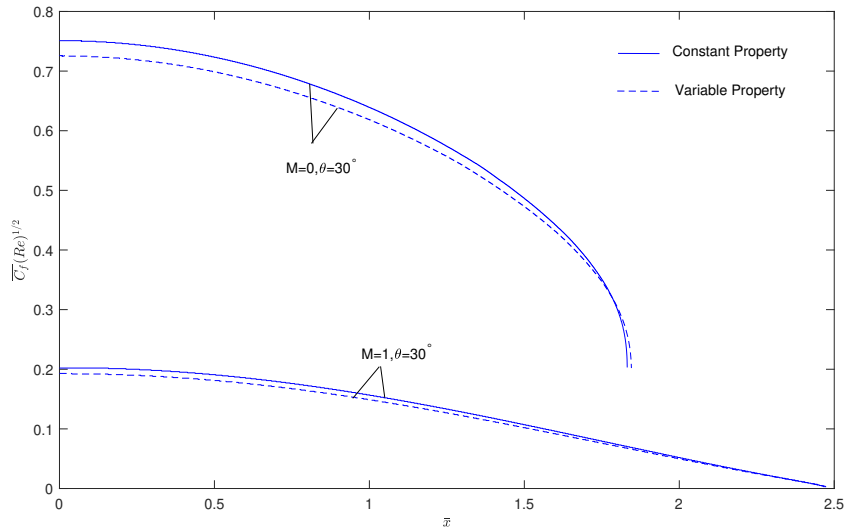

Fig. 5 Effect of the constant and variable properties on the skin friction coefficient $\left(\overline{C_{f}}(R e)^{1 / 2}\right)$

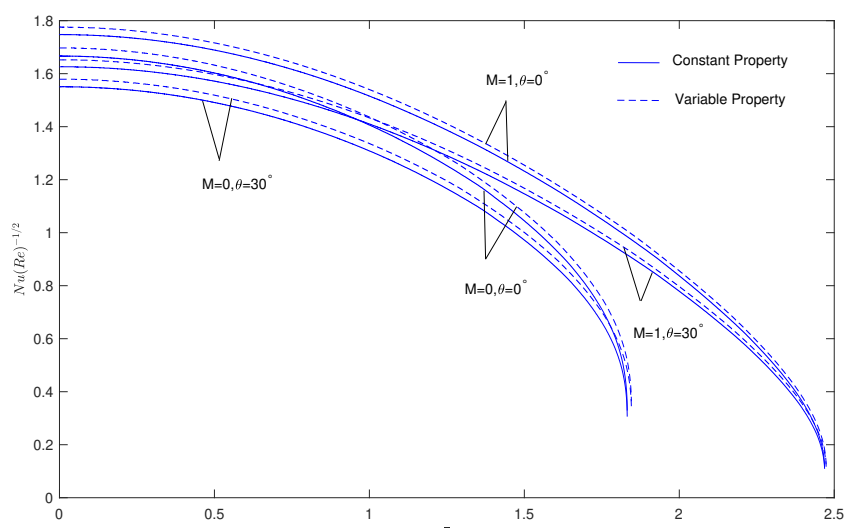

Fig. 6 Effect of the constant and variable properties on the heat transfer coefficient

$$
\left(N S_{\eta}\right)_{\eta}+f S_{\eta}-M P S=2 Q(\bar{x})\left(F S_{\bar{x}}-f_{\bar{x}} S_{\eta}\right)
$$




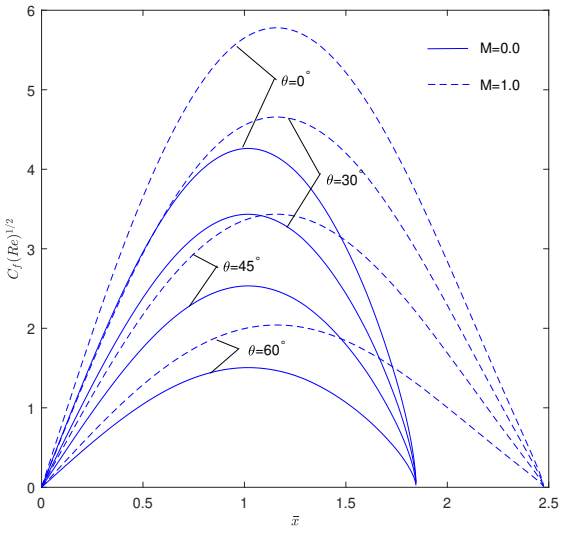

Fig. 7 Effect of the MHD parameter $M$ and yaw angle $\theta$ on the skin friction coefficient $\left(C_{f}(R e)^{1 / 2}\right)$ for variable viscosity and Prandtl number

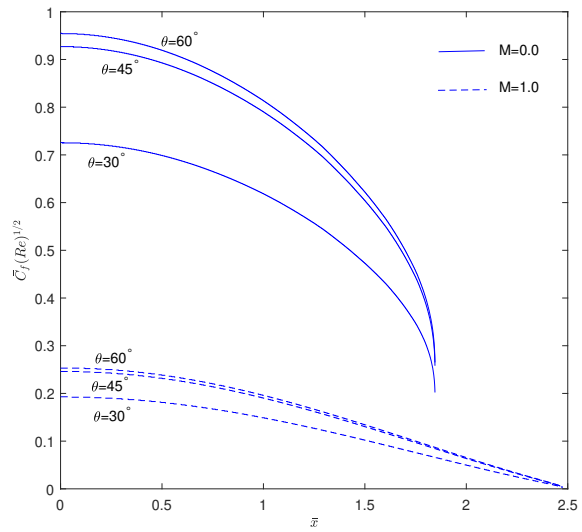

Fig. 8 Effect of the MHD parameter $M$ and yaw angle $\theta$ on the skin friction coefficient $\left(\overline{C_{f}}(R e)^{1 / 2}\right)$ for variable viscosity and Prandtl number

$$
\begin{gathered}
\left(\frac{1}{P r} N G_{\eta}\right)_{\eta}+f G_{\eta}+N E c\left[\left(\frac{u_{e}}{u_{\infty}}\right)^{2} \cos ^{2} \theta F_{\eta}^{2}+\sin ^{2} \theta S_{\eta}^{2}\right] \\
+P E c M\left(\frac{u_{e}}{u_{\infty}}\right)^{2} F(F-1)=2 Q(\bar{x})\left(F G_{\bar{x}}-f_{\bar{x}} G_{\eta}\right)
\end{gathered}
$$

The boundary conditions become

$$
\begin{aligned}
F(\bar{x}, 0) & =0, & F(\bar{x}, \infty) & =1 \\
S(\bar{x}, 0) & =0, & S(\bar{x}, \infty) & =1 \\
G(\bar{x}, 0) & =1, & G(\bar{x}, \infty) & =0
\end{aligned}
$$

The skin friction coefficients in the $x, z$-directions and the heat transfer coefficient can be written as

$$
\begin{gathered}
C_{f}(R e)^{1 / 2}=4 P_{2} P_{1}^{1 / 2}(\cos \theta)^{3 / 2} N_{w}\left(F_{\eta}\right)_{w} \\
\overline{C_{f}}(R e)^{1 / 2}=2^{3 / 2} \cos \left(\frac{\bar{x}}{2}\right) \sqrt{\cos \theta} \sin \theta N_{w}\left(S_{\eta}\right)_{w} \\
N u(R e)^{-1 / 2}=-\sqrt{2 \cos \theta} \cos \left(\frac{\bar{x}}{2}\right)\left(G_{\eta}\right)_{w}
\end{gathered}
$$

where,

$$
\begin{gathered}
C_{f}=\frac{2\left[\mu\left(\frac{\partial u}{\partial y}\right)\right]_{w}}{\rho U_{\infty}^{2}}, \quad \overline{C_{f}}=\frac{2\left[\mu\left(\frac{\partial w}{\partial y}\right)\right]_{w}}{\rho U_{\infty}^{2}} \\
N_{w}=\frac{1}{1+E_{1} G_{w}}=\text { constant }, \quad N u=\frac{R\left(\frac{\partial T}{\partial y}\right)_{w}}{\left(T_{\infty}-T_{w}\right)}
\end{gathered}
$$

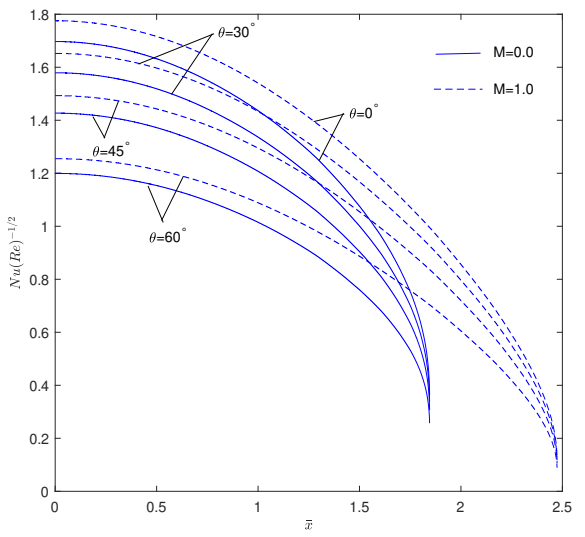

Fig. 9 Effect of the MHD parameter $M$ and yaw angle $\theta$ on the heat transfer coefficient for variable viscosity and Prandtl number

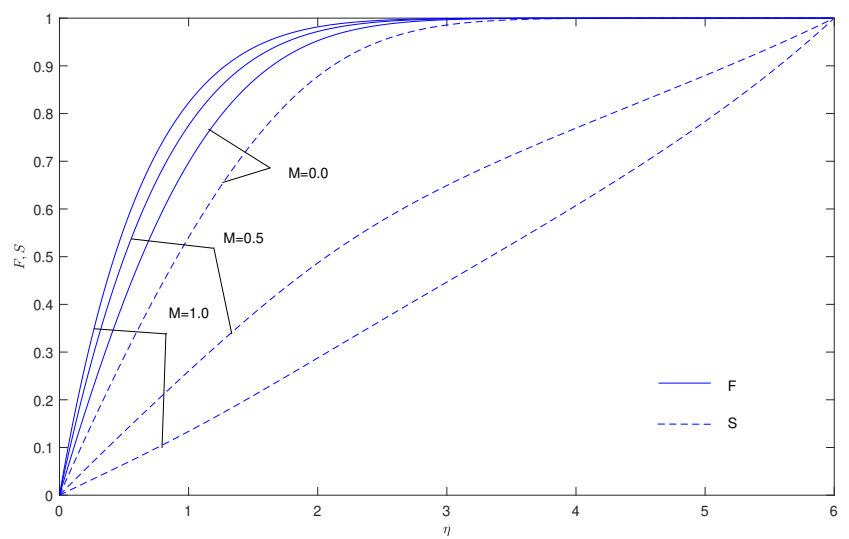

Fig. 10 Effect of the MHD parameter $M$ on the velocity profiles $F, S$ for $\theta=30^{\circ}$, variable viscosity and Prandtl number

\section{RESULTS AND DISCUSSIONS}

The quasi-linearization method is utilised to linearise the non-linear PDEs (20), (21) and (22) and the resulting sequence is, in turn, converted into a block tridiagonal system of algebraic equations (Inouye and Tate, 1974). Finally, Varga's algorithm is implemented to solve the block tridiagonal system (Varga, 2000). The convergence of solution is supposed to be achieved when $\max \left\{\left|\left(F_{\eta}\right)_{w}^{(k+1)}-\left(F_{\eta}\right)_{w}^{(k)}\right|,\left|\left(S_{\eta}\right)_{w}^{(k+1)}-\left(S_{\eta}\right)_{w}^{(k)}\right|,\left|\left(G_{\eta}\right)_{w}^{(k+1)}-\left(G_{\eta}\right)_{w}^{(k)}\right|\right\}<10^{-4}$

The step sizes in $\eta$ and $\bar{x}$ directions respetively are $\Delta \eta=10^{-2}$ and $\Delta \bar{x}=5 \times 10^{-4}$. Here, $\eta_{\infty}$ is taken as 6.0.

The efficiency of our results are verified by comparing the nonsimilar solutions obtained for a horizontal cylinder $\left(\theta=0^{\circ}\right)$ with Sathyakrishna et al. (2001) for $A=0, E c=0, t^{*}=0.0, M=0,0.5,1$ and for a yawed cylinder $\left(\theta=30^{\circ}\right)$ with Revathi et al. (2014) for $E c=0, t^{*}=$ $0.0, M=0, A=0.25,0.0,-0.1$ are presented in Fig. 2 and 3. The results are found to be in excellent agreement.

Figures 4-6 depict the variations of the skin friction coefficients $\left(C_{f}(R e)^{1 / 2}, \overline{C_{f}}(R e)^{1 / 2}\right)$ and the heat transfer coefficient $\left(N u(R e)^{-1 / 2}\right)$ due to constant and variable fluid properties. Irrespective of the MHD parameter $M$ and yaw angle $\theta$, the variable properties diminish $C_{f}(R e)^{1 / 2}$ and $\overline{C_{f}}(R e)^{1 / 2}$ as compared to the constant fluid properties. Contrastingly, the heat transfer is enhanced by the variable fluid properties.

From Figs. 7-9, it is observed that for each $M$ and $\theta, C_{f}(R e)^{1 / 2}$ enhances from zero, hits its maximum and then declines to zero whereas 


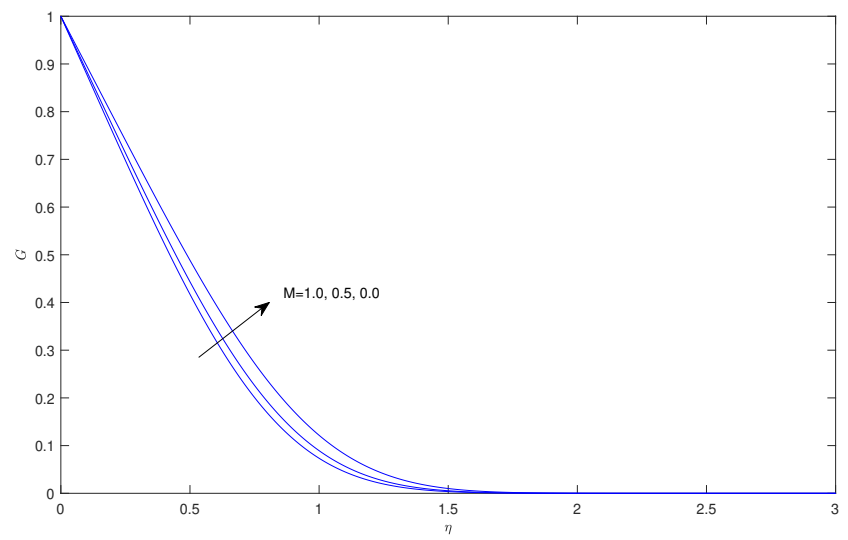

Fig. 11 Effect of the MHD parameter $M$ on the temperature profile $G$ for $\theta=30^{\circ}$, variable viscosity and Prandtl number

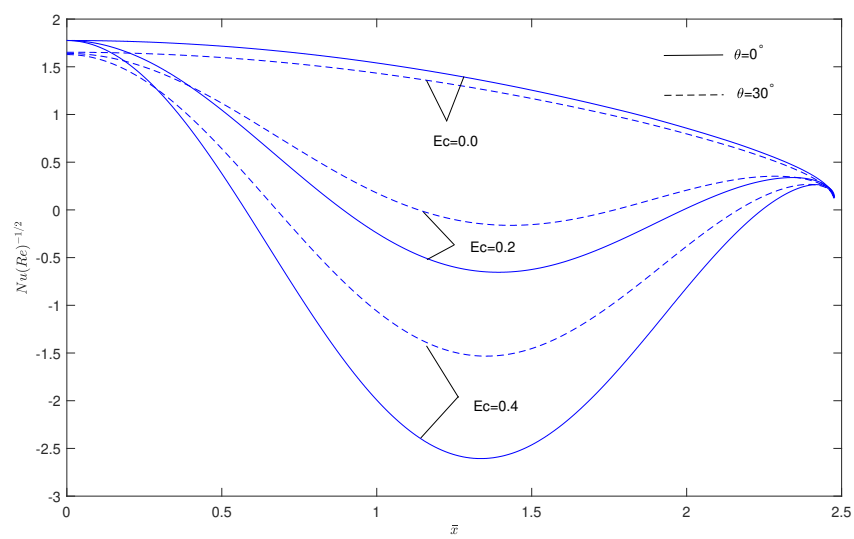

Fig. 12 Effects of the viscous dissipation parameter $E c$ and yaw angle $\theta$ on the heat transfer coefficient for $M=1$, variable viscosity and Prandtl number

$\overline{C_{f}}(R e)^{1 / 2}, N u(R e)^{-1 / 2}$ decline monotonically as $\bar{x}$ further increases. With the enhancing of magnetic effect, $C_{f}(R e)^{1 / 2}$ and $N u(R e)^{-1 / 2}$ increase, but $\overline{C_{f}}(R e)^{1 / 2}$ decreases. However, for a constant magnetic effect $M$, the yaw angle enhancement reverses the above effects. As $M$ changes from 0 to 1 , the point of zero skin friction in the $x$-direction moves downstream. This observation reveals that the separation can be slowed down by enhancing the MHD parameter $M$.

The variations in the velocity profiles in the $x, z$-direction $(F, S)$ and on the temperature profile $(G)$ with respect to $M$ for $\theta=30^{\circ}$ are presented in Figs. 10 and 11. $F$ is reduced with the enhancing of the magnetic effect. However, the effect is just the opposite on $S$ and $G$. This is because the imposed magnetic field generates a supporting force in $x$-direction and an opposing force in $z$-direction. This results in accelerating and decelerating the flow in the respective directions. The impact of $M$ is not remarkable on the temperature profile.

The influence of viscous dissipation parameter $(E c)$ and the yaw angle $(\theta)$ on $N u(R e)^{-1 / 2}$ and $G$ are shown in Figs. 12 and 13. It is noticed that an increment of $E c$ enhances the temperature of the fluid within the boundary layer. For $E c>0$, the fluid's temperature near the wall elevates higher than $T_{w}$, owing to viscous dissipation. This results in the temperature profile surpassing 1 near the body's surface, which then declines to zero.

Figure 12 depicts that $N u(R e)^{-1 / 2}$ declines with an increase of $E c$. The heat transfer parameter becomes negative for $E c>0$, showing the

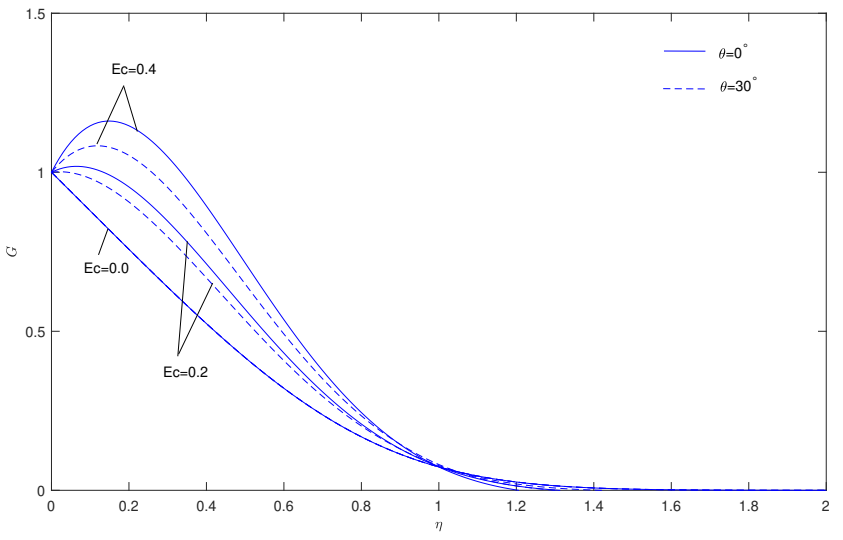

Fig. 13 Effects of the viscous dissipation parameter $E c$ and yaw angle $\theta$ on the temerature profile for $M=1$, variable viscosity and Prandtl number

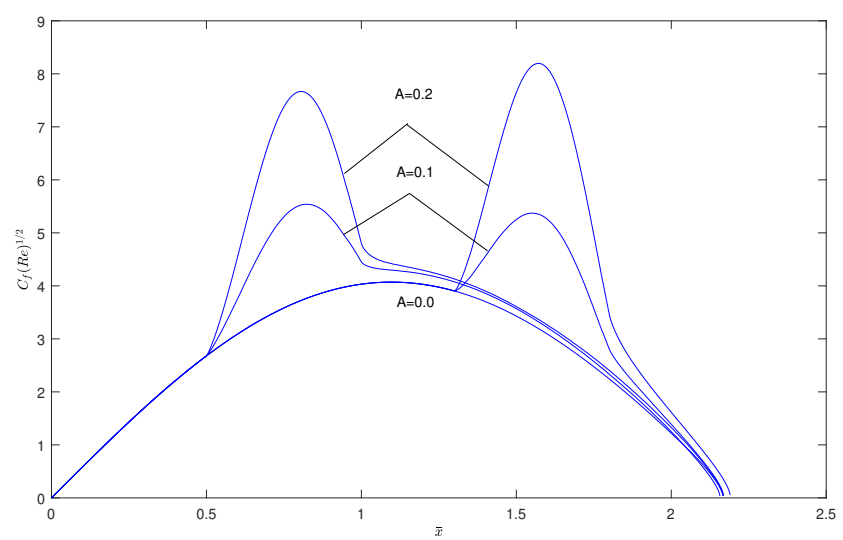

Fig. 14 Effects of suction $(A>0)$ and slot location $\left(\bar{x}_{0}=0.5,1.3\right)$ on the skin friction coefficient $\left(C_{f}(R e)^{1 / 2}\right)$ for $M=0.5$, variable viscosity and Prandtl number

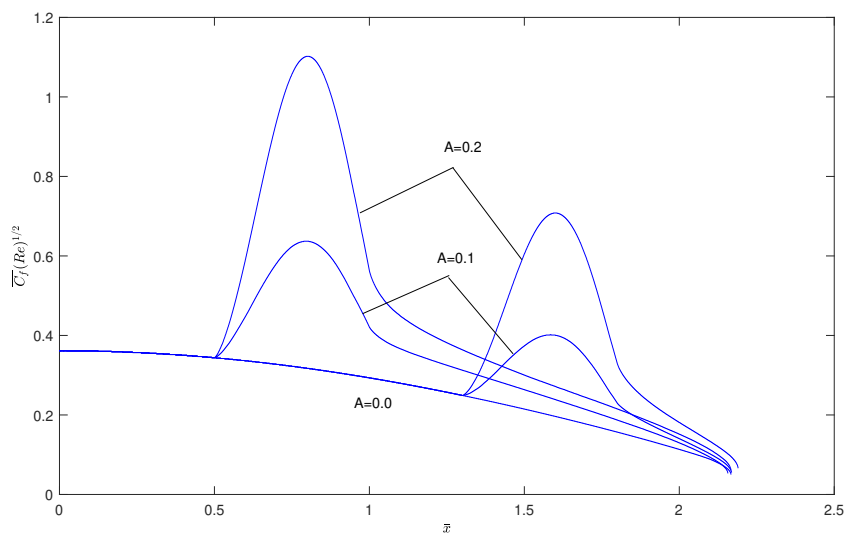

Fig. 15 Effects of suction $(A>0)$ and slot location $\left(\bar{x}_{0}=0.5,1.3\right)$ on the skin friction coefficient $\left(\overline{C_{f}}(R e)^{1 / 2}\right)$ for $M=0.5$, variable viscosity and Prandtl number

inversion of the heat transfer direction. However, this is not observable for $E c=0$. The viscous dissipation parameter has a shallow impact on 


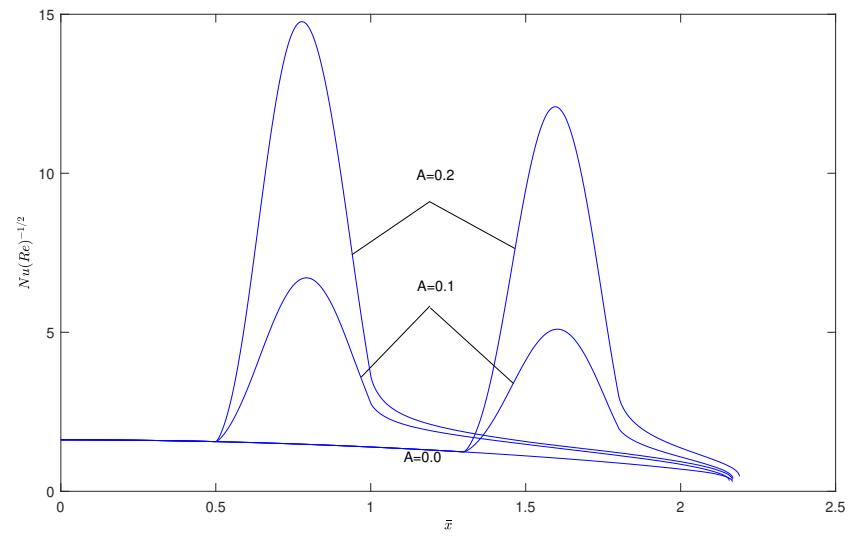

Fig. 16 Effects of suction $(A>0)$ and slot location $\left(\bar{x}_{0}=0.5,1.3\right)$ on the heat transfer coefficient for $M=0.5$, variable viscosity and Prandtl number

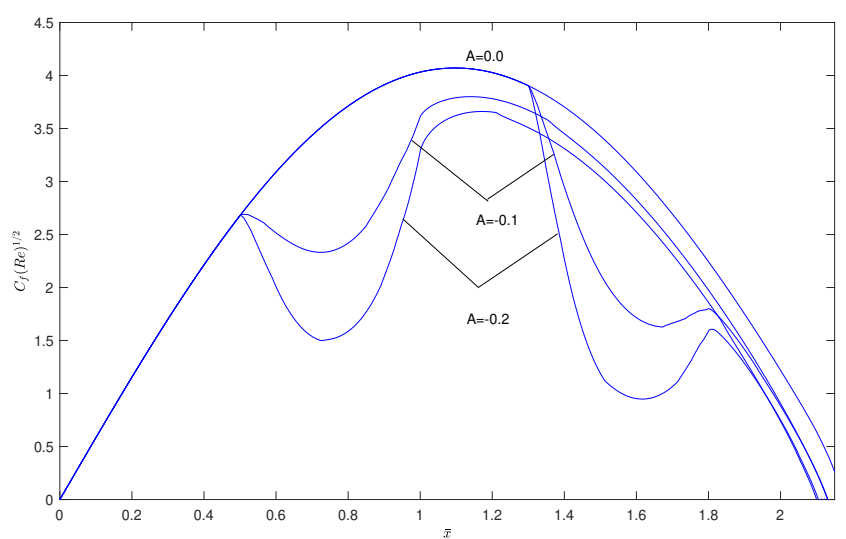

Fig. 17 Effects of injection $(A<0)$ and slot location $\left(\bar{x}_{0}=0.5,1.3\right)$ on the skin friction coefficient $\left(C_{f}(R e)^{1 / 2}\right)$ for $M=0.5$, variable viscosity and Prandtl number

the skin friction coefficients, and hence the corresponding figures are not presented here.

The impact of the mass transfer through two different slots $[0.5,1.0]$ and $[1.3,1.8]$ on $C_{f}(R e)^{1 / 2}, \overline{C_{f}}(R e)^{1 / 2}$ and $N u(R e)^{-1 / 2}$ are shown in Figs. 14-19. For slot suction $(A>0)$, as the slot starts, $C_{f}(R e)^{1 / 2}$, $\overline{C_{f}}(R e)^{1 / 2}$ and $N u(R e)^{-1 / 2}$ increase and hit their maximum before reaching its end. After that $\overline{C_{f}}(R e)^{1 / 2}$ and $N u(R e)^{-1 / 2}$ decline and reach a non-zero finite value, but $C_{f}(R e)^{1 / 2}$ almost vanishes. The injection $(A<0)$ shows the opposite effect. The reason behind this is the function $v_{w}$ considered in Eq. (17), which describes the effect of mass transfer within a slot. The term $\sin \left(\omega^{*}\left(\bar{x}-\bar{x}_{0}\right)\right)$ in $v_{w}$ increases in the first half of the slot, reaches its highest value of 1 at $\left(\bar{x}_{0}+\bar{x}_{0}^{*}\right) / 2$ and decreases to 0 afterwards. Hence, the corresponding changed velocity field influences the temperature field, leading to a change in skin friction and heat transfer coefficients. It is observed that a small increment in the suction parameter $A>0$ influences the zero skin friction in $x$-direction to move downstream and hence delays the separation. This phenomenon is much noticeable if the location of the slot $[0.5,1.0]$ is also moved downstream to $[1.3,1.8]$. However, in the case of injection, increasing the injection parameter $A<0$ and moving the slot location downstream makes the zero skin friction in $x$-direction to move upstream.

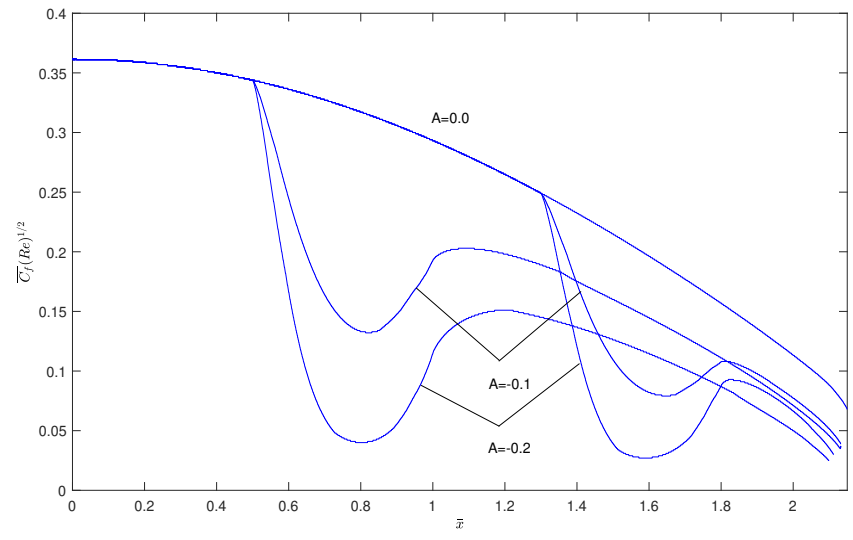

Fig. 18 Effects of injection $(A<0)$ and slot location $\left(\bar{x}_{0}=0.5,1.3\right)$ on the skin friction coefficient $\left(\overline{C_{f}}(R e)^{1 / 2}\right)$ for $M=0.5$, variable viscosity and Prandtl number

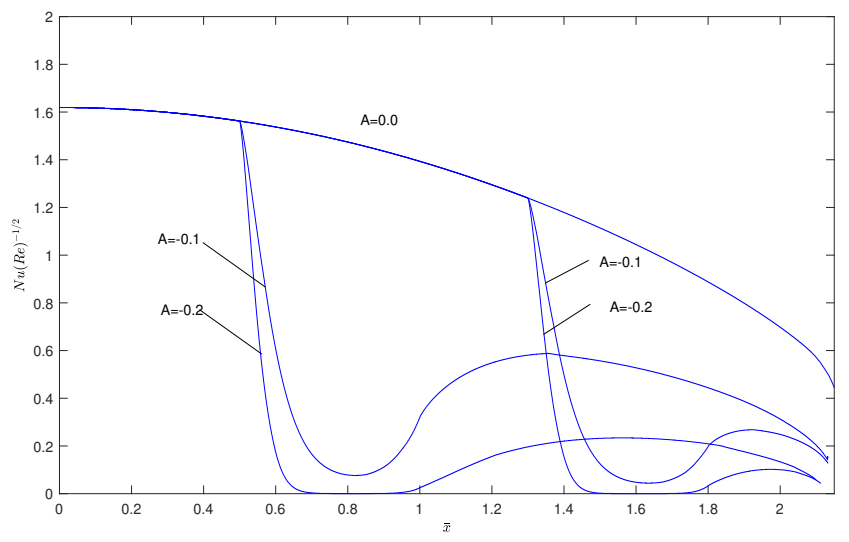

Fig. 19 Effects of injection $(A<0)$ and slot location $\left(\bar{x}_{0}=0.5,1.3\right)$ on the heat transfer coefficient for $M=0.5$, variable viscosity and Prandtl number

\section{CONCLUSIONS}

The steady MHD flow problem over a yawed cylinder has been solved numerically, and the following observations are made:

- The separation can be slowed down by enhancing the MHD parameter $M$.

- With an increase of yaw angle $\theta$, the skin friction coefficient $C_{f}(R e)^{1 / 2}$ and the heat transfer coefficient $N u(R e)^{-1 / 2}$ declines irrespective of the value of $M$.

- The enhancement of viscous dissipation effect $E c$ enhances the temperature $(G)$ of the fluid within the boundary layer while increasing the yaw angle $\theta$ diminishes $G$.

- Overshoot in the temperature profile is observed near the body surface for $E c>0$.

- Increasing suction $(A>0)$ and the slot's movement in downstream direction moves the zero skin friction in $x$-direction downstream, whereas the injection's effect is the opposite.

\section{NOMENCLATURE}

A Dimensionless mass transfer parameter

$B_{0} \quad$ Magnetic field strength 


\begin{tabular}{|c|c|}
\hline$c_{p}$ & Specific heat $(\mathrm{kJ} / \mathrm{kg} \cdot \mathrm{K})$ \\
\hline$C_{f}, \overline{C_{f}}$ & Skin friction coefficient in $x, z$-directions, respectively \\
\hline$E c$ & Eckert number (viscous dissipation parameter) \\
\hline$f_{w}(x)$ & Surface mass transfer distribution \\
\hline$f$ & Dimensionless stream function \\
\hline$F$ & Dimensionless velocity component in the $x$-direction \\
\hline$G$ & Dimensionless temperature \\
\hline$k$ & Thermal conductivity $(\mathrm{W} / \mathrm{m} \cdot \mathrm{K})$ \\
\hline$M$ & MHD parameter \\
\hline$N u$ & Nusselt number \\
\hline$N$ & $\frac{\mu}{\mu}$ Viscosity ratio \\
\hline $\operatorname{Pr}$ & Prandtl number \\
\hline$R e$ & Reynolds number \\
\hline$R$ & Radius of the yawed cylinder \\
\hline$S$ & Dimensionless velocity component in the $z$-direction \\
\hline$T$ & Temperature $(\mathrm{K})$ \\
\hline$u, v, w$ & $\begin{array}{l}\text { Dimensional velocity components in } x, y, z \text {-directions, } \\
\text { respectively }(\mathrm{m} / \mathrm{s})\end{array}$ \\
\hline$U$ & Steady state velocity at the boundary layer's edge \\
\hline$x, y, z$ & $\begin{array}{l}\text { Dimensional meridional, azimuthal and normal distances, } \\
\text { respectively }(\mathrm{m})\end{array}$ \\
\hline $\bar{x}$ & Dimensionless meridional distance \\
\hline $\bar{x}_{0}, \bar{x}_{0}^{*}$ & Ends of slot \\
\hline \multicolumn{2}{|c|}{ Greek Symbols } \\
\hline$\beta(\xi)$ & Pressure gradient \\
\hline$\eta, \xi$ & Transformed coordinates \\
\hline$\Delta \eta, \Delta \bar{x}$ & Step sizes in $\eta-, \bar{x}-$ directions, respectively \\
\hline$\mu$ & Dynamic viscosity $(\mathrm{Kg} / \mathrm{m} \cdot \mathrm{s})$ \\
\hline$\nu$ & Kinematic viscosity $\left(\mathrm{m}^{2} / \mathrm{s}\right)$ \\
\hline$\rho$ & Density $\left(\mathrm{Kg} / \mathrm{m}^{3}\right)$ \\
\hline$\sigma$ & Electrical conduction \\
\hline$\theta$ & Yaw angle \\
\hline$\omega^{*}$ & Slot length parameter \\
\hline$\psi$ & Dimensional stream function $\left(\mathrm{m}^{2} / \mathrm{s}\right)$ \\
\hline \multicolumn{2}{|c|}{ Subscripts } \\
\hline$e$ & Conditions at the edge of the boundary layer \\
\hline$w$ & Conditions at the surface of the sphere \\
\hline$\infty$ & Conditions in the free stream \\
\hline
\end{tabular}

\section{REFERENCES}

Aldoss, T.K., Ali, Y.D., and Al-Nimr, M.A., 1996, "MHD Mixed Convection from a Horizontal Circular Cylinder," Numerical Heat Transfer, Part A: Applications, 30(4), 379-396.

http://dx.doi.org/10.1080/10407789608913846.

Chin, K.E., Nazar, R., Arifin, N.M., and Pop, I., 2007, "Effect of Variable Viscosity on Mixed Convection Boundary Layer Flow over a Vertical Surface Embedded in a Porous Medium," International Communications in Heat and Mass Transfer, 34(4), 464-473.

http://dx.doi.org/10.1016/j.icheatmasstransfer.2007.01.002.

Davies, T., and Walker, G., 1977, "On Solutions of the Compressible Laminar Boundary-Layer Equations and their Behaviour near Separation," Journal of Fluid Mechanics, 80(2), 279-292.

http://dx.doi.org/10.1017/S0022112077001670.

Dewey, C.F., and Gross, J.F., 1967, "Exact Similar Solutions of the Laminar Boundary-Layer Equations," Advances in Heat Transfer, vol. 4, 317446, Elsevier.

http://dx.doi.org/10.1016/S0065-2717(08)70276-4.

Eisenhuth, J.J., and Hoffman, G.H., 1981, "Wall Temperature Estimation for Heated Underwater Bodies," Journal of Hydronautics, 15(1), 90-96. http://dx.doi.org/10.2514/3.63212.
El-Amin, M.F., 2003, "Combined Effect of Viscous Dissipation and Joule Heating on MHD Forced Convection over a Non-Isothermal Horizontal Cylinder Embedded in a Fluid Saturated Porous Medium," Journal of Magnetism and Magnetic Materials, 263(3), 337-343. http://dx.doi.org/10.1016/S0304-8853(03)00109-4.

Eswara, A.T., and Nath, G., 1994, "Unsteady Nonsimilar TwoDimensional and Axisymmetric Water Boundary Layers with Variable Viscosity and Prandtl Number," International Journal of Engineering Science, 32(2), 267-279.

http://dx.doi.org/10.1016/0020-7225(94)90006-X.

Ganapathirao, M., Chamkha, A.J., and Rao, N.S., 2019, "Effects of Non-Uniform Slot Suction/Injection and Chemical Reaction on Mixed Convective MHD Flow along a Vertical Wedge Embedded in a Porous Medium," Frontiers in Heat and Mass Transfer, 13(15), 1-13. http://dx.doi.org/10.5098/hmt.13.15.

Inouye, K., and Tate, A., 1974, "Finite-Difference Version of QuasiLinearization Applied to Boundary-Layer Equations," AIAA Journal, 12(4), 558-560. http://dx.doi.org/10.2514/3.49286.

Jenifer, A.S., Saikrishnan, P., and Lewis, R.W., 2021, "Unsteady MHD Mixed Convection Flow of Water over a Sphere with Mass Transfer," Journal of applied and computational mechanics, 7(2), 935-943. https://doi.org/10.22055/JACM.2021.35920.2761.

King, R., 1977, "Vortex Excited Oscillations of Yawed Circular Cylinders," Journal of Fluids Engineering, 99(3), 495-501. http://dx.doi.org/10.1115/1.3448825.

Kumar, V.S.S., Pai, N.P., and Devaki, B., 2021, "Analysis of MHD Flow and Heat Transfer of Laminar Flow between Porous Disks," Frontiers in Heat and Mass Transfer, 16(3), 1-7. http://dx.doi.org/10.5098/hmt.16.3.

Marshall, J.S., 2003, "Wake Dynamics of a Yawed Cylinder," Journal of Fluids Engineering, 125(1), 97-103. http://dx.doi.org/10.1115/1.1523069.

Mityakov, A., Mityakov, V., Sapozhnikov, S., Gusakov, A., Bashkatov, A., Seroshtanov, V., Zainullina, E., and Babich, A., 2017, "Hydrodynamics and Heat Transfer of Yawed Circular Cylinder," International Journal of Heat and Mass Transfer, 115(A), 333-339.

https://doi.org/10.1016/j.ijheatmasstransfer.2017.07.055.

Mohammad, S.A., 2020, "Effects of Variable Viscosity on Heat and Mass Transfer by MHD Mixed Convection Flow along a Vertical Cylinder Embedded in a Non-Darcy Porous medium," Frontiers in Heat and Mass Transfer, 14(7), 1-10.

http://dx.doi.org/10.5098/hmt.5.4.

Nagaraju, G., Garvandha, M., and Murthy, J.V.R., 2019, "MHD Flow in a Circular Horizontal Pipe under Heat Source/Sink with Suction/Injection on Wall," Frontiers in Heat and Mass Transfer, 13(6), 1-8.

http://dx.doi.org/10.5098/hmt.13.6.

Pantokratoras, A., 2005, "Forced and Mixed Convection Boundary Layer Flow along a Flat Plate with Variable Viscosity and Variable Prandtl Number: New Results," Heat and Mass Transfer, 41(12), 1085-1094. http://dx.doi.org/10.1007/s00231-005-0627-8.

Patil, P.M., Shashikant, A., Roy, S., and Hiremath, P.S., 2020, "Mixed Convection Flow past a Yawed Cylinder," International Communications in Heat and Mass Transfer, 114, 104582.

http://dx.doi.org/10.1016/j.icheatmasstransfer.2020.104582. 
Ponnaiah, S., 2012, "Boundary Layer Flow over a Yawed Cylinder with Variable Viscosity: Role of Non-Uniform Double Slot Suction (Injection)," International Journal of Numerical Methods for Heat \& Fluid Flow, 22(3), 342-356.

http://dx.doi.org/10.1108/09615531211208051.

Ramberg, S.E., 1983, "The Effects of Yaw and Finite Length upon the Vortex Wakes of Stationary and Vibrating Circular Cylinders," Journal of Fluid Mechanics, 128(1), 81-107.

http://dx.doi.org/10.1017/S0022112083000397.

Revathi, G., Saikrishnan, P., and Chamkha, A., 2014, "Non-Similar Solutions for Unsteady Flow over a Yawed Cylinder with Non-Uniform Mass Transfer through a Slot," Ain Shams Engineering Journal, 5(4), 11991206.

http://dx.doi.org/10.1016/j.asej.2014.04.009.

Roy, S., 2001, "Non-Uniform Mass Transfer or Wall Enthalpy into a Compressible Flow over Yawed Cylinder," International Journal of Heat and Mass Transfer, 44(16), 3017-3024.

http://dx.doi.org/10.1016/S0017-9310(00)00355-0.

Roy, S., and Saikrishnan, P., 2004, "Non-Uniform Slot Injection (Suction) into Water Boundary Layer Flow past Yawed Cylinder," International Journal of Engineering Science, 42(19), 2147-2157.

http://dx.doi.org/10.1016/j.ijengsci.2003.12.008.

Saikrishnan, P., and Roy, S., 2003a, "Non-Uniform Slot Injection (Suction) into Steady Laminar Water Boundary Layer Flow over a Rotating Sphere," International Journal of Heat and Mass Transfer, 46(18), 33893396.

http://dx.doi.org/10.1016/S0017-9310(03)00137-6.

Saikrishnan, P., and Roy, S., 2003b, "Non-Uniform Slot Injection (Suction) into Water Boundary Layers over (i) a Cylinder and (ii) a Sphere,"
International Journal of Engineering Science, 41(12), 1351-1365. http://dx.doi.org/10.1016/S0020-7225(03)00043-0.

Sathyakrishna, M., Roy, S., and Nath, G., 2001, "Unsteady TwoDimensional and Axisymmetric MHD Boundary-Layer Flows," Acta Mechanica, 150(1), 67-77. http://dx.doi.org/10.1007/BF01178545.

Subhashini, S.V., and Samuel, N., 2016, "Non-Similar Solution of a Steady Compressible Boundary Layer Flow over a Thin Cylinder," Frontiers in Heat and Mass Transfer, 7(29), 1-7.

http://dx.doi.org/10.5098/hmt.7.29.

Tashtoush, B., Kodah, Z., and Al-Ghasem, A., 2000, "Heat Transfer Analysis of a Non-Newtonian Fluid on a Power-law Stretched Surface with Suction or Injection for Uniform and Cooled Surface Temperature," International Journal of Numerical Methods for Heat \& Fluid Flow, 10(4), 385-396.

http://dx.doi.org/10.1108/09615530010327378.

Thakur, A., Liu, X., and Marshall, J.S., 2004, "Wake Flow of Single and Multiple Yawed Cylinders," Journal of Fluids Engineering, 126(5), 861870.

http://dx.doi.org/10.1115/1.1792276.

Varga, S., 2000, Matrix Iterative Analysis, 2nd ed., Springer-Verlag Berlin Heidelberg, Germany.

Venkatachala, B.J., and Nath, G., 1980, "Nonsimilar Laminar Incompressible Boundary Layers with Vectored Mass Transfer," Proceedings of the Indian Academy of Sciences Section C: Engineering Sciences, 3(2), 129-142.

http://dx.doi.org/10.1007/BF02842902. 